\title{
Constrains on particle acceleration in Rosette and Orion nebulae with Fermi-LAT observations
}

\author{
Fabien Krayzel, Gilles Maurin, Giovanni Lamanna* \\ LAPP, Université Savoie Mont Blanc, CNRS/IN2P3, 9 Chemin de Bellevue, 74941 \\ Annecy-le-Vieux, France \\ E-mail: Fabien.Krayzel@lapp.in2p3.fr, Gilles.Maurin.in2p3.fr, \\ giovanni.lamanna@lapp.in2p3.fr
}

\section{Nukri Komin}

School of Physics, University of the Witwatersrand, 1 Jan Smuts Avenue, Braamfontein, Johannesburg, 2050 South Africa.

E-mail: nukri.komin@wits.ac.za

\author{
Alexandre Marcowith \\ LUPM, Université de Montpellier II, CNRS/IN2P3, Place Eugène Bataillon, 34095 Montpellier, \\ France \\ E-mail: Alexandre.Marcowitheuniv-montp2.fr
}

\begin{abstract}
The Rosette and Orion nebulae are two young massive star clusters in which no supernova explosion has occurred yet. They are therefore good candidates to look for non thermal radiation originated by particle acceleration mechanisms taking place within stellar-wind bubbles (i.e. induced by the collective effects of stellar winds). The gamma-ray emission from these nebulae as observed by the Fermi-LAT together with prediction from a phenomenological model have been considered in this work. Upper limits on the fraction of mechanical energy converted into accelerated particles have been obtained. Finally the expected TeV Îş-ray emission, which could be explored in the future with the advent of new generation gamma-ray observatories such as CTA, has been also considered..
\end{abstract}

The 34th International Cosmic Ray Conference,

30 July- 6 August, 2015

The Hague, The Netherlands

\footnotetext{
* Speaker.
} 


\section{Introduction}

The shells of supernova remnants (SNRs) are considered as the main acceleration sites of Galactic Cosmic Rays. The discovery of synchrotron radiation from SNR shells already proves the acceleration of electrons up to relativistic energies. The $\mathrm{TeV} \gamma$-ray emission detected from these shells (for a review of the $\mathrm{TeV}$-detected SNRs see e.g. [1]), further supports the hypothesis of SNRs being sources of Galactic Cosmic Rays. This emission can be interpreted either as Inverse Compton up-scattering of low-energy photons by relativistic electrons or as a product of decaying pions produced by hadronic interactions of Cosmic Rays.

Similarly to SNRs, Cosmic Rays can also be accelerated in the shells of superbubbles. These superbubbles are blown into the interstellar medium by the combined stellar winds of associations of massive stars and successive supernova explosions. So far, $\mathrm{GeV}$ and $\mathrm{TeV} \gamma$-ray emissions were detected from two superbubbles: Cygnus OB2 in the Milky Way [2] by the Fermi-LAT and 30 Dor $\mathrm{C}$ in the Large Magellanic Cloud [3] by the H.E.S.S. system. The $\gamma$-ray emission is evidence for Cosmic Ray particle acceleration in theses superbubbles. In both superbubbles several supernova explosions have surely occurred and it is possible that supernova remnants are responsible for the particle acceleration.

Gamma-ray emission from star clusters and stellar-wind bubbles (SWBs) where no supernova explosion have occurred yet would be the best evidence for particle acceleration originated by stellar winds interactions.

\section{Astrophysical properties of two embedded star clusters}

In this work we present the search for $\gamma$-ray emission from the Rosette and Orion nebulae. The properties of the two objects are summarised in Table 1.

The Rosette Nebula is a HII region ionized by the embedded star cluster NGC 2244 in the Monoceros constellation. This cluster is a part of the Mon OB2 association. It creates a bubble of rarefied matter in the interstellar medium thanks to the mechanical energy released by the most massive stars. The most massive one is HD 46223, which is an O4(f) V type. The whole cluster releases more than $10^{37} \mathrm{erg} . \mathrm{s}^{-1}$.

The Trapezium cluster in the Orion constellation ionizes the HII region of the Orion Nebula. This cluster is a part of the Ori OB1d association. The mechanical luminosity released by the most massive stars like Ori C (O7 V type) is $10^{36} \mathrm{erg} . \mathrm{s}^{-1}$.

\begin{tabular}{lccccccc}
\hline \hline Cluster & O stars & $\begin{array}{c}\text { Distance } \\
(\mathrm{kpc})\end{array}$ & $\begin{array}{c}\text { Luminosity } \\
\left(\mathrm{erg} . \mathrm{s}^{-1}\right)\end{array}$ & $\begin{array}{c}\text { Age } \\
(\mathrm{Myr})\end{array}$ & $\begin{array}{c}\text { Density } \\
\left(\mathrm{cm}^{-3}\right)\end{array}$ & $\begin{array}{c}\text { Radius } \\
(\mathrm{pc})\end{array}$ & $\begin{array}{c}\text { Angular size } \\
\left({ }^{\circ}\right)\end{array}$ \\
\hline \hline NGC 2244 & 6 & 1.6 & $1.1 \times 10^{37}$ & 1.9 & 15 & 16.9 & 1.3 \\
Trapezium & 2 & 0.4 & $1.2 \times 10^{36}$ & 2.5 & 8900 & 3.7 & 1.0 \\
\hline \hline
\end{tabular}

Table 1: Astrophysical properties of the Rosette and Orion nebulae.

The stellar wind luminosity of each cluster has been estimated from the number of O-type stars and standard model quantifying the stellar wind loss of massive stars $[4,5]$. Neither of the clusters supernova remnants, pulsars or pulsar wind nebulae were detected therein. Further on, the 
clusters do not exhibit any stars beyond the main-sequence (like Wolf-Rayet stars). Therefore it is very likely, that no supernova explosion has occurred yet.

\section{Fermi-LAT data analysis}

The Large Area Telescope (LAT) on board the Fermi satellite is a pair conversion telescope sensitive to photons with energies from $20 \mathrm{MeV}$ to more than $300 \mathrm{GeV}$. For the analysis presented here we used data which were recorded between 2008 August 4 and 2014 August 8 and which are reconstructed with pass 7 reconstruction. The data were analysed using Fermi ScienceTools V9r32p5 with the python interface enrico [6].

Events of the "source" class falling within a circular region of interest (ROI) of $10^{\circ}$ radius centred on the source position were selected. Events with zenith angles of more than $100^{\circ}$ and time intervals when the rocking angle was larger than $52^{\circ}$ have been rejected. In order to reduce the background and investigate the $\mathrm{GeV}$ regime a minimum energy threshold of $3 \mathrm{GeV}$ was considered.

The potential $\gamma$-ray emission of the SWB was modelled as a diffuse source corresponding to a projected sphere with their HII region radius and a power law spectrum. The background model includes all $\gamma$-ray sources from the third Fermi LAT source catalog (3FGL, see [7]) as well as the Galactic diffuse emission (gll_iem_v05_rev1) and the isotropic background (iso_source_v05). The normalisation parameters of the diffuse background components were kept free in the fit. An unbinned maximum-likelihood fit (using gt like) was performed using the P7_REPSOURCE_V15 instrument response functions (IRFs).

\subsection{NGC 2244 - Rosette nebula}

The energy threshold of $3 \mathrm{GeV}$ is crucial here in order to avoid low energy $\gamma$-emission produced by the shock from the supernova remnant Monoceros Loop which is interacting with the HII region of the Rosette nebula [8]. The background model includes 4 point-like sources within $3^{\circ}$ of NGC 2244 where the parameters were kept free, and additional 18 point-like sources with fixed spectral parameters.

The left panel of Fig. 3.1 shows the test statistics (TS) map after the fit. The maximumlikelihood on NGC 2244 results in a TS of 5.17. In the absence of a significant signal, an upper limit on the $\gamma$-ray flux was extracted with a Bayesian calculation (Integral method). Assuming a power law with an index $\Gamma=-2.25$, the upper limit on the integrated photon flux is set to $\Phi_{\gamma}<$ $2.50 \times 10^{-10} \mathrm{~cm}^{-2} \mathrm{~s}^{-1}$ at $95 \%$ confidence level.

\subsection{NGC 1976 - Orion nebula}

The background model consists of 3 point sources within $3^{\circ}$ of NGC 1976 with free parameters and another 15 point-like sources with fixed spectral parameters. The energy threshold was set to $1 \mathrm{GeV}$.

The right panel of Fig. 3.1 shows the test statistics (TS) map in the direction of the Orion nebula after the fit. The maximum-likelihood on NGC 1976 results in a TS of 5.0. Assuming a power law with an index $\Gamma=-2.25$, the upper limit on the integrated photon flux is set to $\Phi_{\gamma}<$ $3.40 \times 10^{-10} \mathrm{~cm}^{-2} \mathrm{~s}^{-1}$ at $95 \%$ confidence level. 

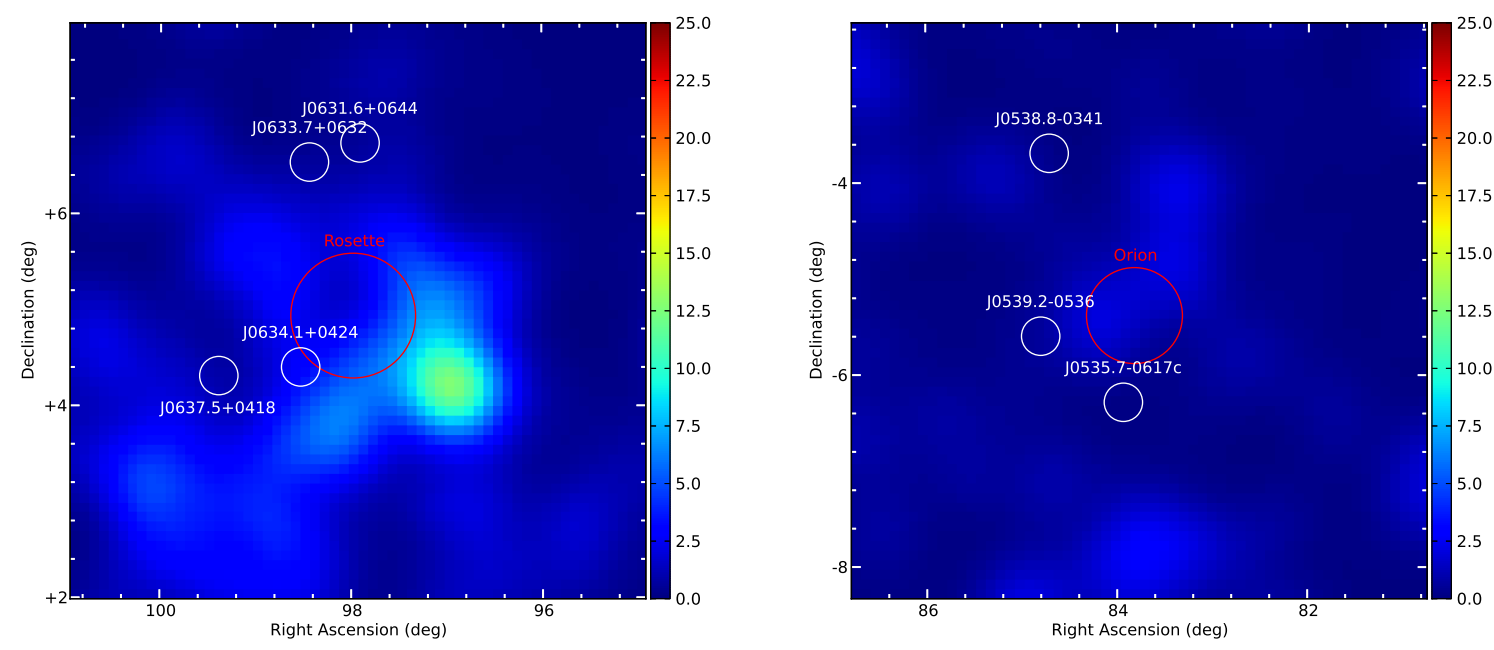

Figure 1: Test statistics (TS) maps of the Rosette Nebula (left panel) and the Orion nebula (right panel). The green circle denotes the region of interest of the bubble, white circles represent sources from the 3FGL catalogue.

\section{Discussion}

We compare the upper limit on the $\gamma$-ray flux with predictions from a one-zone model where gamma-ray radiations are produced by cluster-accelerated particles interacting with the matter in dense surrounding HII regions. This model takes into account Synchrotron radiation, Inverse Compton emission and Bremsstrahlung of accelerated leptons as well as $\gamma$-ray production through pion decay in hadronic interactions of accelerated protons with the surrounding molecular cloud. The details of this model are presented at this conference in a separate contribution [9]. The main parameter in this model is the fraction $\xi$ of the stellar wind luminosity which is converted into energetic particles. The magnetic field for the synchrotron emission is a priori not known for these objects. As detailed in [10], the mean magnetic field inside HII regions would be a function of the mean density. The density is set to $15 \mathrm{~cm}^{-3}$ for the Rosette Nebula [11] and $8900 \mathrm{~cm}^{-3}$ for the Orion Nebula [12] (see Table 1), justifying the choice of $10 \mu \mathrm{G}$ for the Rosette Nebula and $100 \mu \mathrm{G}$ for the Orion Nebula.

Figure 2 the expected emission from the consideration of the most energetic models which result to be still compatible with the experimental upper limit on the $\mathrm{GeV} \gamma$-ray spectral density obtained here from the analysis of Fermi-LAT data.

It can be seen that emission from accelerated protons dominates emission from relativistic leptons, mainly due to the high target density in the HII region. The model allows the derivation of an upper limit on the fraction $\xi$ which is $2.7 \times 10^{-2}$ for the Rosette Nebula and $6.4 \times 10^{-2}$ for the Orion Nebula. The results of this work are summarised in Table 2. At this limit the future Cherenkov Telescope Array (CTA) will be able to detect $\gamma$-ray emission from these objects. In 


\begin{tabular}{llll}
\hline \hline Cluster & TS & $\Phi_{U L}^{95 \%}\left(\mathrm{~cm}^{-2} \mathrm{~s}^{-1}\right)$ & $\xi_{U L}^{95 \%}$ \\
\hline \hline NGC 2244 & 5.17 & $2.50 \times 10^{-10}$ & $2.7 \times 10^{-2}$ \\
NGC 1976 & 5.0 & $3.4 \times 10^{-10}$ & $6.4 \times 10^{-2}$ \\
\hline \hline
\end{tabular}

Table 2: Summary of the results. TS is the test statistic value for $\gamma$-ray emission at the position of the superbubbles and $\Phi_{U L}^{95 \%}$ is the upper limit on the $\gamma$-ray flux. $\xi_{U L}^{95 \%}$ is the upper limit on the fraction $\xi$ of the stellar wind luminosity $L_{w}$ released by the central cluster which is converted into energetic particles.

Figure 2 such a perspective is considered when 50h CTA observation time is considered for each source.

\section{Summary}

We searched for evidence for Cosmic Ray accelerations through stellar winds in young stellar clusters. We selected two stellar clusters, NGC 2244 in the Rosette Nebula and NGC 1976 in the Orion Nebula, which exhibit massive O-type stars and and which have not yet shown any supernova explosions. We extracted upper limits on the $\gamma$-ray flux from archival Fermi-LAT data.

Using a one-zone model for the particle acceleration in the stellar cluster and the interaction in the surrounding model and scanning all model parameters in realistic boundaries, we get an upper limit on the fraction $\xi$ of the conversion of stellar wind power of the cluster into accelerated particles to $2.7 \times 10^{-2}$ for the Rosette Nebula and $6.4 \times 10^{-2}$ for the Orion Nebula. The Cherenkov Telescope Array (CTA) could probe the efficiencies $\xi$ below the percent.

The analysis presented here will be repeated with the recently released pass 8 reconstructed Fermi-LAT data. This study will be extended to a larger sample of young massive star clusters.

\section{References}

[1] N. Komin and for the H. E. S. S. collaboration, Search for Galactic Cosmic Ray Sources with H.E.S.S, in Particle Physics and Cosmology (L. Celnikier, J. Dumarchez, B. Klima, and Jean Tran Than Van, eds.), 2011. ArXiv e-prints 1201.0641.

[2] M. Ackermann, M. Ajello, A. Allafort, L. Baldini, J. Ballet, G. Barbiellini, D. Bastieri, A. Belfiore, R. Bellazzini, B. Berenji, et al., A cocoon of freshly accelerated cosmic rays detected by fermi in the cygnus superbubble, science 334 (2011), no. 6059 1103-1107.

[3] HESS Collaboration, A. Abramowski et al., The exceptionally powerful TeV gamma-ray emitters in the Large Magellanic Cloud, Science 347 (2015), no. 6220 406-412, [arXiv: 1501.0657].

[4] J. Puls, J. S. Vink, and F. Najarro, Mass loss from hot massive stars, A\&A Rev. 16 (Dec., 2008) 209-325, [arXiv:0811.0487].

[5] A. Sternberg, T. L. Hoffmann, and A. W. A. Pauldrach, Ionizing Photon Emission Rates from O- and Early B-Type Stars and Clusters, ApJ 599 (Dec., 2003) 1333-1343, [astro-ph/0312232].

[6] D. A. Sanchez and C. Deil, Enrico : a Python package to simplify Fermi-LAT analysis, ArXiv e-prints (July, 2013) [arXiv:1307.4534].

[7] The Fermi-LAT Collaboration, Fermi Large Area Telescope Third Source Catalog, ArXiv e-prints (Jan., 2015) [arXiv:1501.0200]. 

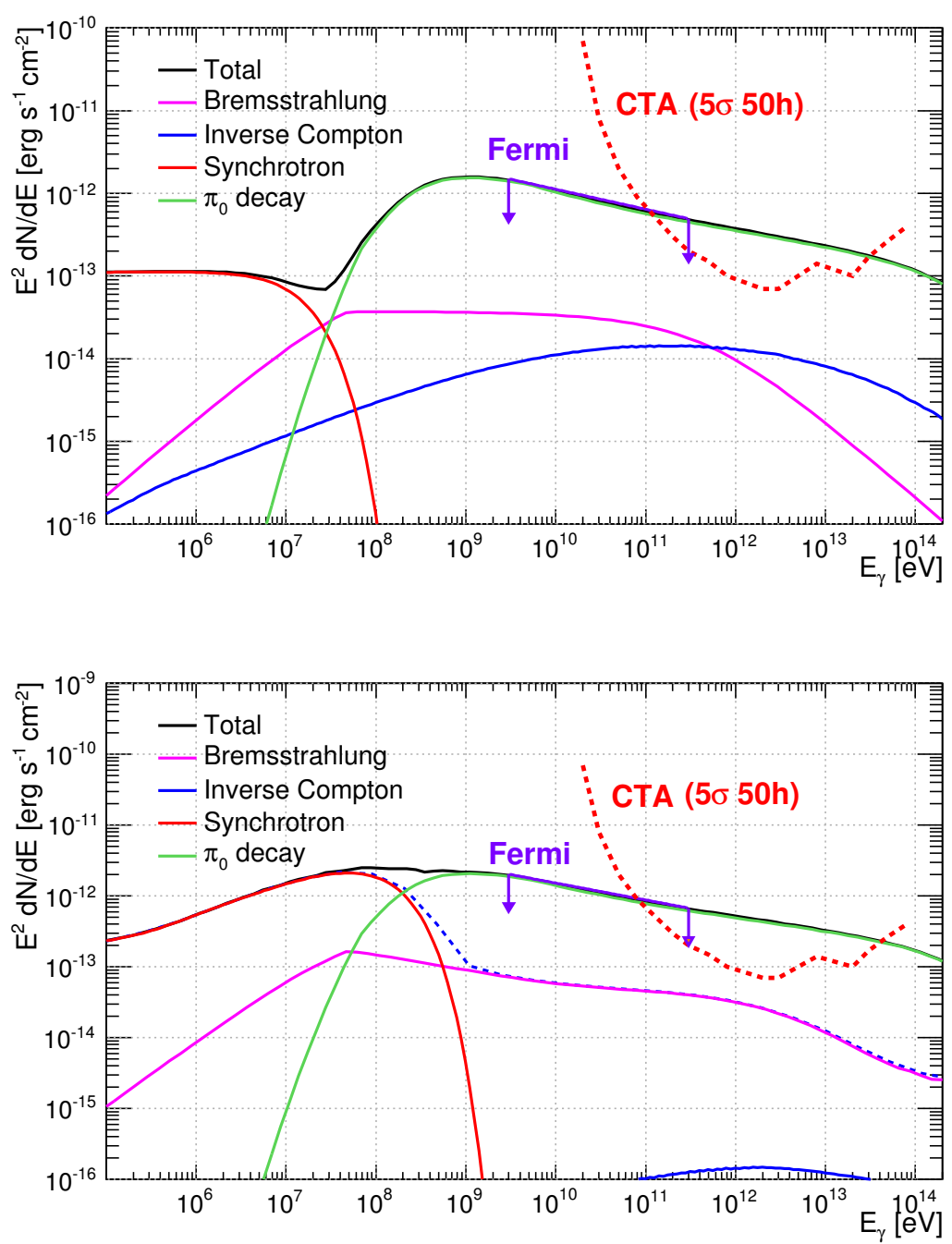

Figure 2: Fermi-LAT upper limits and theoretical expectations on the spectral energy density deduced by models of the Rosette Nebula (top panel) and Orion Nebula (bottom panel) for realistic parameters (the ratio $K_{e p}$ between accelerated electrons and protons set to $10^{-2}$, a magnetic field turbulence level $\delta B / B$ fixed to 0.1 and its index $v$ set to $3 / 2$ ). The model takes into account particle acceleration in the star cluster and the interaction of the particles with the surrounding material. The model details are presented in a separate contribution [9]. The magnetic field for the synchrotron emission is $10 \mu \mathrm{G}$ for the Rosette Nebula and $100 \mu \mathrm{G}$ for the Orion Nebula. The density of the surrounding medium is set to $15 \mathrm{~cm}^{-3}$ for the Rosette Nebula and $8900 \mathrm{~cm}^{-3}$. The red dashed line represents the sensitivity of the future Cherenkov Telescope Array (CTA) (5 $\sigma$ detection in $50 \mathrm{~h},[13])$. 
[8] H. Katagiri and S. Sugiyama, Fermi-LAT Study of Gamma-ray Emission in the Direction of the Monoceros Loop Supernova Remnant, in 40th COSPAR Scientific Assembly, vol. 40 of COSPAR Meeting, p. 1419, 2014.

[9] F. Krayzel, A. Marcowith, G. Maurin, N. Komin, and G. Lamanna, Phenomenological description of young massive star clusters, in ICRC, 2017. no. 77.

[10] L. Harvey-Smith, G. J. Madsen, and B. M. Gaensler, Magnetic fields in large-diameter h ii regions revealed by the faraday rotation of compact extragalactic radio sources, The Astrophysical Journal 736 (2011), no. 283.

[11] F. Bruhweiler, R. F. Ferrero, M. Bourdin, and T. Gull, The young interstellar bubble within the rosette nebula, The Astrophysical Journal 719 (2010), no. 21872.

[12] C. Esteban, M. Peimbert, J. García-Rojas, M. Ruiz, A. Peimbert, and M. Rodriguez, A reappraisal of the chemical composition of the orion nebula based on very large telescope echelle spectrophotometry, Monthly Notices of the Royal Astronomical Society 355 (2004), no. 1 229-247.

[13] Y. Becherini, B. Khélifi, S. Pita, M. Punch, and CTA Consortium, Advanced analysis and event reconstruction for the CTA Observatory, in American Institute of Physics Conference Series (F. A. Aharonian, W. Hofmann, and F. M. Rieger, eds.), vol. 1505 of American Institute of Physics Conference Series, pp. 769-772, Dec., 2012. arXiv:1211.5997. 